\title{
Tractional Maculopathies in Age-related Macular Degeneration
}

\author{
Cynthia X Qian, ${ }^{1}$ William J Foster ${ }^{2,3,4}$ and Flavio A Rezende ${ }^{1,5}$ \\ 1. Chief Resident, Department of Ophthalmology, University of Montreal, Quebec, Canada; 2. Associate Professor, Department of Ophthalmology, Weill-Cornell \\ Medical College at The Methodist Hospital, Houston, US; 3. Associate Professor, Department of Physics, The University of Houston, Texas, US; 4. Associate \\ Professor, The Methodist Hospital Research Institute, Houston, Texas, US; 5. Associate Professor, Department of Ophthalmology, University of Montreal,
}

Quebec, Canada; 6. Associate Professor, Pontifícia Universidade Católica, Rio de Janeiro, Brazil

\begin{abstract}
Age-related macular degeneration (AMD) is the leading cause of blindness among the elderly in developed countries. Much progress has been and continues to be made in search of better visual outcomes for dry and exudative AMD. Over the past decade, the importance of vitreomacular attachments has been recognised in AMD. In this article, the authors better characterise and describe vitreomacular and photoreceptor-retinal pigment epithelium interface relationships in AMD among treated and untreated patients and describe the surgical options available as well as their outcomes and possible complications.
\end{abstract}

\section{Keywords}

Age-related macular degeneration, tractional maculopathy, posterior vitreous detachment, macular hole, epiretinal membrane, Müller cells, reactive gliosis, vitreoretinal adhesion, vitreomacular traction syndrome

Disclosure: This work did not receive funding from any source. None of the authors have any proprietary or financial interest in the products discussed in this article.

Received: 14 November 2011 Accepted: 13 December 2011 Citation: European Ophthalmic Review, 2012;6(5):300-6 DOI: 10.17925/EOR.2012.06.05.300 Correspondence: Flavio A Rezende, Department of Ophthalmology, University of Montreal, Montreal, QC, Canada. E: frezendef@hotmail.com

Age-related macular degeneration (AMD) is an insidious progressive loss of visual function that occurs with ageing and is the leading cause of visual loss in the Western Hemisphere in the elderly. ${ }^{12}$ Although common, the pathways mediating its onset and progression are complex and multifactorial. While many studies have looked at the pathogenesis and physiological changes that occur at the outer retina during AMD, research on inner retinal changes is less common. More specifically, when AMD is compounded by degenerative tractional changes at the vitreomacular interface and inner retina, the problem becomes greater than the sum of its parts. In this article, the authors address the different aetiologies of vitreomacular interface pathologies found in association with both dry and exudative AMD, their pathogenesis, and how their pharmacological and surgical treatment responses differ from the presentation and treatment of either condition alone.

\section{Vitreomacular Adhesions}

Vitreomacular adhesion (VMA) defines a condition in which the vitreous gel and posterior hyaloid are abnormally adherent to the retina. When a posterior vitreous detachment (PVD) is incomplete and does not undergo a normal synchronous sequence of synchisis and syneresis, a taut anterior/posterior traction on the underlying macula can be created. ${ }^{3,4}$ This leads to vitreomacular traction (VMT) and subsequent visual degradation, now identifiable and characterisable on a more regular basis through advances in time- and spectral-domain optical coherence tomography (OCT) imaging. ${ }^{5.6}$ More commonly, traction can lead to a spectrum of retinal architectural distortions, ranging from cystoid macular oedema, epiretinal membrane (ERM), and macular holes up to macular and retinal pigment epithelium (RPE) detachments. ${ }^{\text {? }}$

\section{Vitreomacular Adhesions and Age-related Macular Degeneration}

The current prevalence of tractional maculopathy is estimated to be $6.4 \%$ within the American population over the age of 50 . Recent data have corroborated previous ultrasound findings that incomplete abnormal PVD is more common than was previously thought and that associated posterior VMA seems to be more common among patients with AMD than in age-matched controls, placing prevalence at 22-36 \%. ${ }^{8-11}$ In particular, this seems to be the case more often in advanced exudative cases than in patients with non-exudative AMD. ${ }^{8,10-13}$ Frequently, vitreous attachment sites to the macula correspond to areas of choroidal neovascularisation (CNV), suggesting a direct relationship.,8 Mojana and colleagues imaged VMA in AMD, showing a $27.8 \%$ prevalence in exudative cases and $24.5 \%$ in non-exudative cases. In this study, traction was identified on OCT in 59 and $13 \%$ of patients in each group, respectively, showing a strong direct correlation between adhesion and traction. ${ }^{12}$ These abnormal vitreomacular interactions in AMD seem to be present in different populations in the world. In a cohort of Brazilian patients of 204 eyes with AMD imaged with time-domain OCT, $16.7 \%$ had VMT, $7.8 \%$ had ERM, $1 \%$ had macular holes and $15.2 \%$ had incomplete perifoveal PVD. ${ }^{14}$ When using spectral domain OCT imaging, the number of patients with VMA identified may increase up to $73 \% .{ }^{15}$ Given that patients with AMD have a higher likelihood of partial PVD, it is often difficult to tell whether their visual symptoms and intra- or subretinal fluid are more likely to be related to the tractional component of VMA or to the anatomical distortion caused by the degenerative component of $A M D$, with the fortuitous discovery of a concurrent partial PVD (see 
Figures 1 and 2). Current knowledge does not indicate whether VMA in AMD is a cause or a result of AMD pathophysiology (see Figure 3). Several authors believe that although tractional maculopathy is not sufficient to induce AMD, it nonetheless has a key role in the natural history of AMD. ${ }^{16}$

Patients with dry AMD and tractional maculopathy can go on to develop exudative AMD through the direct tractional effects on the retina and the RPE. This may lead to a chronic state of low-grade inflammation and ischaemia resulting from the obstruction of oxygen and nutrient diffusion, and an increase in pro-inflammatory and angiogenic growth factor load in the macular region, which can precede CNV (see Figure 4), 9,12,17,18 In addition, a partial PVD leaves vitreous cortical remnants on the inner limiting membrane (ILM), which might later serve as a scaffold for cellular proliferation and traction..$^{19}$ Therefore, understanding how these tractional forces arise and countering their evolution may be paramount in preventing the inexorable progression of AMD.

\section{Müller Cells and Reactive Gliosis}

A first step in developing a better understanding of VMA in AMD pathogenesis is to understand the changes at the cellular level that are different from those that occur in normal eyes. Although the end cascade event is always the loss of photoreceptors and RPE through different pathways of genetic, chemical or photic insults in accordance with other authors, the authors believe that Müller cells have a major role upstream in concurrent VMA and AMD..$^{20-22}$

Müller cells and astrocytes comprise the macroglia of the retina. In the retina, Müller cells far outnumber glial astrocytes as the main support cells. ${ }^{23}$ In fact, only Müller cells are present in the normal foveolar centre. The Müller cells span all neurosensorial retinal layers, enabling them to provide an architectural scaffold that is essential for neuronal cell orientation, communication and information processing, to regulate ion concentrations, to limit the spread of cytotoxic neurotransmitters and to have a role in glycolysis and lactate metabolism. ${ }^{23-25}$

Müller cells respond to damage to the retina in a graded manner depending on the extent and severity of the insult. 26,27 This response is known as 'reactive gliosis'. Its primary function is one of neuroprotection through conservative gliosis, where chronic inflammation and the disruption of the RPE sets in, possibly activated through the recruitment of human leukocyte antigen (HLA) antigens, the complement cascade, microglia and circulating macrophages. ${ }^{28,29}$ After initial moderate retinal stress, Müller cells are directly involved in the signal transduction of stress, doing so by releasing cytokines and vasoactive substrates such as vascular endothelial growth factor (VEGF), pigment epithelium-derived factor (PEDF), transforming growth factor (TGF)- $B$ and activating extracellular signal-regulated kinases (ERKs), an early response to injury. 2,30-34

When the insult signal overwhelms the system, Müller cells undergo massive gliosis. ${ }^{22,35-38}$ They then contribute detrimentally to neuronal cell death through the excitotoxic pathway and dysregulated $\mathrm{K}+$ conductance. In addition, they become reactive and re-enter the cell cycle to hypertrophy, forming gliotic scars such as the disciform scars seen in late exudative AMD (see Figure 5). ${ }^{39}$ Intermediate filament protein glial fibrillary acidic proteins (GFAP) within Muller cells, a universal hallmark of retinal injury, are also upregulated. ${ }^{38,40}$

\section{Figure 1: Spectral-domain optical Coherence} Tomography in Partial Posterior Vitreous Detachment overlying Exudative Age-related Macular Degeneration with Retinal Pigment Epithelium Detachment

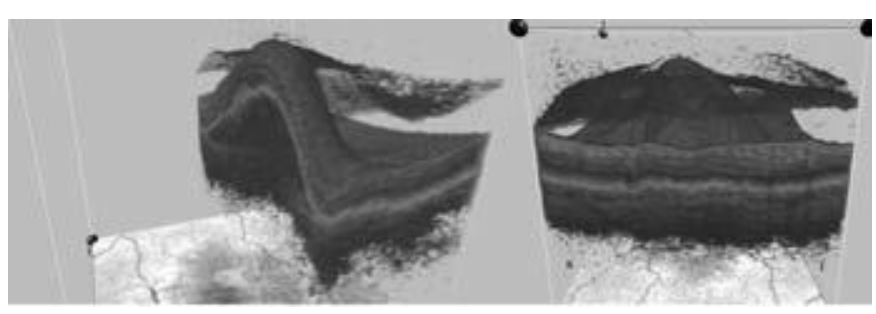

Before spectral-domain optical coherence tomography (OCT), many would call this case a tractional retinal pigment epithelium (RPE) detachment; however, with three-dimensional imaging, it is clearer that it is a partial posterior vitreous detachment (PVD) overlying exudative macular changes without an evident tractional component.

Figure 2: Two-dimensional Spectral-domain Image of Partial Posterior Vitreous Detachment and Active Choroidal Neovascularisation with Sub- and Intraretinal Fluid

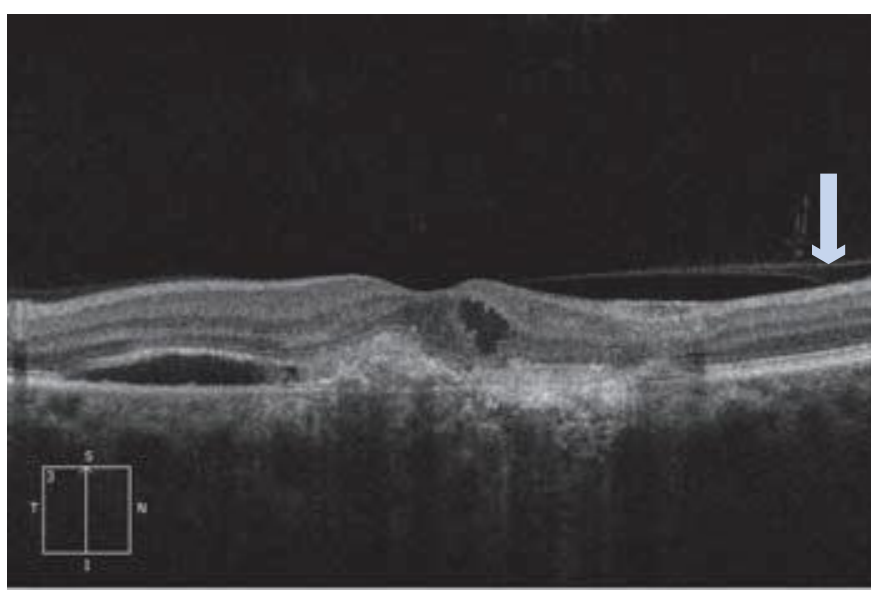

Note the subtle vitreoschisis (arrow)

\section{Figure 3: Dry Age-related Macular Degeneration and Vitreomacular Adhesion}

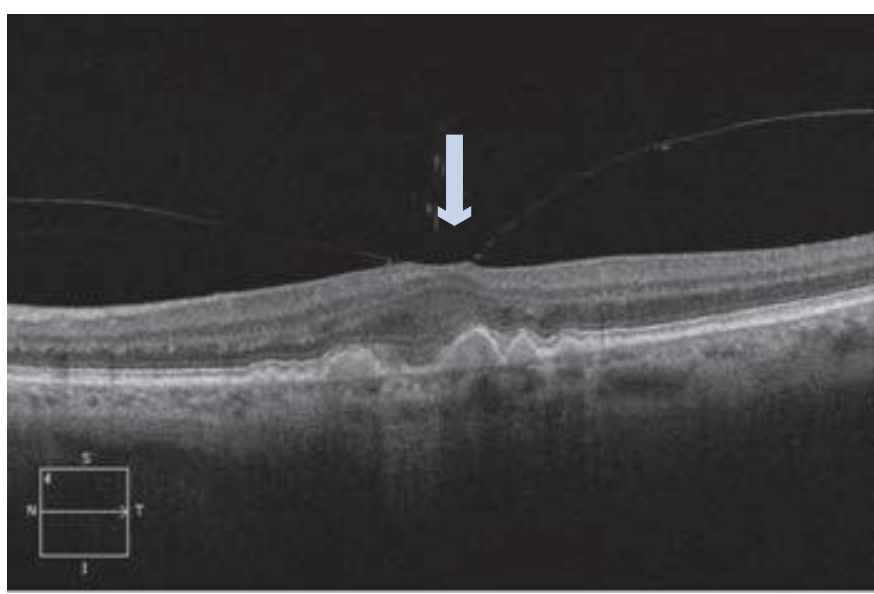

Note the flattening of the foveal center secondary to vitreomacular traction (VMT), but without intra- or subretinal fluid.

In pathological post mortem AMD specimens, GFAP expression was observed to be upregulated directly in regions overlying atrophic or drusenoid RPE changes. Similarly, VMA is also more commonly seen overlying areas of CNV. Both findings might indicate a direct localising relationship between retinal disease and Müller cell reactivity. ${ }^{41-44}$ 
Figure 4: Drusenoid Retinal Pigment Epithelium Detachment with Initial Perifoveal Posterior Vitreous Detachment

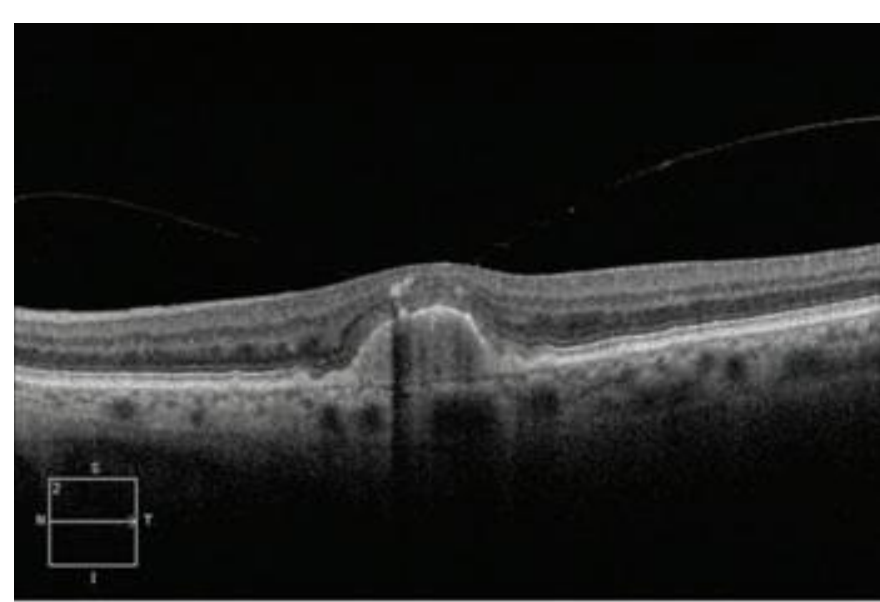

Note the intraretinal hyper-reflective retinal pigment epithelium (RPE) clump showing its disruption, migration and possibly interaction with Müller cells.

Figure 5: Spectral-domain optical coherence Tomography of a Case of Disciform Scar and Epiretinal Membrane in Exudative Age-related Macular Degeneration, a Clinical Expression of Retinal Glial Cells Migration towards the Choroid (Disciform Scar) and the Vitreomacular Interface (Epiretinal Membrane)

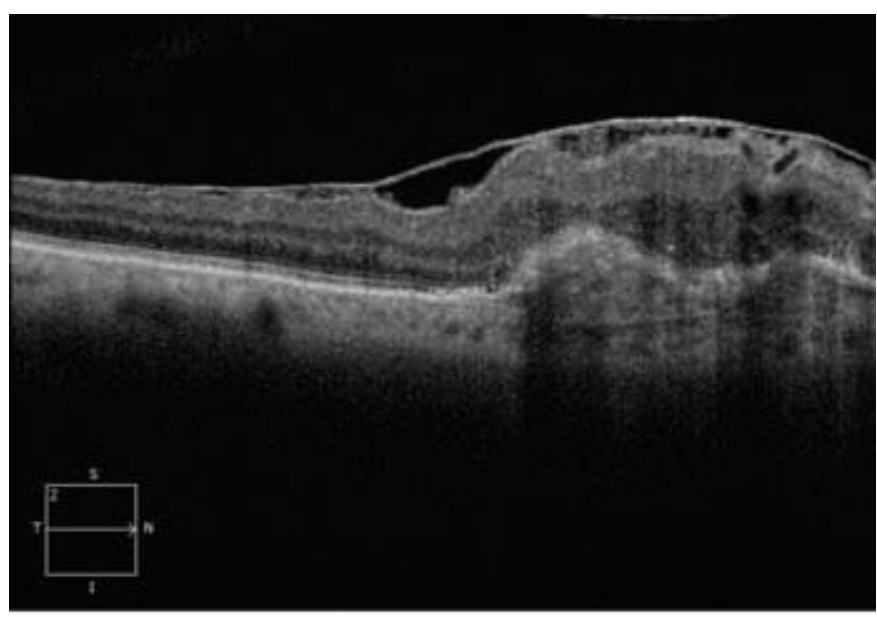

Through GFAP fluorescence, it has already been observed that reactive Müller cells in search of higher oxygen milieu might translocate to both the subretinal space adjacent to photoreceptors and through Bruch's membrane towards the choroid. Alternately, it may extend into the vitreous humor adherent to the vitreous side of the ILM, probably under the influence of local growth factors and cytokines, such as the extracellular matrix-degrading proteolytic matrix metalloproteinases (see Figure 5). 20,21,45-47 Once there, Müller cells can undergo phenotypic changes to express de novo expression of myofibroblast-like characteristics, exhibiting fibrocontractive properties at both the inner and outer retinal layers of their extensions. ${ }^{48,49}$ This has been proven by the incorporation of glial contractile tissue into ERMs, PVR and other tractional structures and by the evidence of Müller cells inducing photoreceptor separation from the RPE similar to a retinal detachment. ${ }^{41}$ The presence of localised macular ischaemia, oxidative stress and chronic inflammation also disturbs the expression of the major potassium channel (Kir4.1) of the Müller cells, with impairment of water transport across these glial cells contributing to macular oedema in addition to vascular leakage..$^{50}$

Thus, the presence of trans-differentiated reactive Müller cells at the vitreomacular and photoreceptor-RPE junction under the influence of vitreous growth factors might be a significant component explaining the frequent association of tractional maculopathy with $\mathrm{AMD}$, as well as the underlying mechanistic forces destabilising the retina during tractional maculopathy surgery in AMD. ${ }^{45}$

\section{Epiretinal Membrane and Age-related Macular Degeneration}

Most ERMS are associated with a high incidence of concurrent complete or partial PVD. ERM in AMD may develop as a result of VMT and inflammation causing ILM dehiscence and enabling migration of reactive Müller cells with myofibroblast transformation and astrocytes from the inner retina to the vitreal side of the ILM (see Figure 6). ${ }^{6,30,51-53}$

Guidry and colleagues have clearly shown that Müller cells in normal retinas are not a component of the overlying ILM but have loose contact points. In a study of 44 peeled human ILM specimens analysed by phase-contrast microscopy and type IV immunofluorescent antibody staining, no Müller cell membrane or axonal components were found attached to the removed ILM. ${ }^{54}$ Another study of ILM-denuded pig retinas showed intact underlying Müller cell membranes. ${ }^{48}$ Both studies confirm that, in a normal eye, the ILM is separate from the underlying retina. In AMD, ultrastructural analyses of ERM with VMT showed that there is incorporation of Müller and glial elements into the ILM, with the presence of a fibrocellular multilayer interposed with vitreous collagen and directly on the ILM with predominant myofibroblasts. ${ }^{45}$ This corroborates the role that Müller and astrocyte glial cells have in reactive gliosis and traction, as previously stated (see Figure 7 ).

\section{Macular Holes and Age-related Macular Degeneration}

Macular holes in AMD arise from the same tractional pathologies that are active in ERM and AMD, and can be considered a progression and more severe clinical manifestation of the VMA forces at work in AMD (see Figure 8). In addition, tangential forces across the gap shown to be related to ERM and newly synthesised collagen fibrils within the cortical vitreous are also involved. ${ }^{55-57}$ Post-mortem specimens of macular holes often show the presence of subtle pre- and epi-retinal membranes in $30-73 \%$ of cases. ${ }^{58}$ This presence is no coincidence and the membranes are thought to evolve from gliotic reactions and to have an essential role in macular hole dynamics. ${ }^{59}$ Perpendicular focal anterior attachments have been shown on OCT, supporting the important role that vitreous traction has in the formation of macular holes.$^{60}$ In addition, the alignment of the perifoveal Müller cells is also perpendicular, suggesting that the macular hole stages, as previously described by Gass, are initiated at the stage I level by inner retina layer Müller cell involvement. ${ }^{6}$ There is possibly an initial inherent inner retinal weakness and pseudocyst formation that is compounded by focal Müller glial cell proliferation in an unsuccessful attempt to repair and bridge the gap, creating secondary traction as cells migrate around the perifoveal denuded ILM.

\section{Management of Age-related Macular Degeneration and Vitreomacular Adhesion}

Given that anomalous and partial PVD might have an important role in AMD progression, prophylactic surgical and pharmacological methods 
of traction release can be considered as an additional part of the treatment for AMD to improve retinal distortion. However, limited data (case reports, small case series, short follow-up) are available on surgical outcomes of tractional maculopathies in patients with AMD. OCT has revolutionised the detection of previously unrecognised cases of VMT and is now an indispensable tool in all cases of combined diagnoses. ${ }^{6}$

\section{Pars Plana Vitrectomy}

Pars plana vitrectomy (PPV) continues to be the mainstay treatment in releasing the traction on the retina induced by vitreomacular adhesions. .11,62 $^{2}$

Some researchers have postulated that PPV, as a form of artificial PVD alone, might be sufficient to simulate its beneficial effects in AMD. Alternately, vitrectomy might be beneficial through the release of tractional damages that ultimately lead to RPE atrophy, the reduction of inflammatory mediators from the milieu and the provision of a higher oxygen diffusion environment in areas that have localised ischaemia. In addition, ILM peeling to remove the scaffold for glial and myofibroblast cell migration into the vitreoretinal interface has also been advocated, as many authors have found this to correlate with higher anatomical and functional success in macular hole and ERM surgery, and lower recurrence rates. ${ }^{57,58,63}$ To facilitate visualisation during surgery, the use of intravitreal vital dyes, such as Trypan blue, triamcinolone acetonide, indocyanine green and more recently brilliant blue, is encouraged for staining the ERM and ILM. However, the risks of using these dyes in the presence of concomitant tractional maculopathies and AMD are unknown and therefore should be used with caution. In addition, endotamponade with an intraocular gas is recommended to ensure long-term surgical success after vitrectomy and membrane peeling for macular holes. ${ }^{64}$

Roller and colleagues recently conducted the first retrospective pilot study of paired eyes in non-exudative AMD patients who underwent vitrectomy in one eye for $\mathrm{MH}$ and ERM while the clinically similar $\mathrm{AMD}$ disease was observed in the fellow eye. Although the sample size was small $(n=22)$, their results demonstrated that vitrectomised eyes did better than fellow eyes and that AMD did not progress. ${ }^{65}$

The authors conducted a prospective interventional small-gauge surgical series between 2005 and 2009 on the management of tractional maculopathy in AMD. In all, 12 patients with dry and exudative AMD were enrolled and followed up for at least 12 months post surgery. Ten patients underwent 25-ga vitrectomy and two patients underwent 27-ga vitrectomy. Two of two eyes with macular holes closed despite CNV reactivation (see Figure 8); four VMTs were operated on without any complications; of the six ERMs, three had no complications, two developed intraoperative RPE tears and one developed a new small subfoveal CNV one month post-operatively (see Figure 9). Overall, our results showed that despite post-operative or new CNV, visual acuity improved in all eyes following small-gauge vitreoretinal surgery for tractional maculopathies in AMD. This was also the first time that intraoperative iatrogenic RPE tears were demonstrated to develop during surgery for ERM in AMD.66

\section{Anti-Vascular Endothelial Growth Factor}

Diseases that might be amenable to anti-VEGF treatment should also receive intravitreal injections first, as a concurrent vitrectomy might decrease the vitreous half-lives of such agents. Both ranibizumab
Figure 6: Epiretinal Membrane in Exudative and Dry Age-related Macular Degeneration

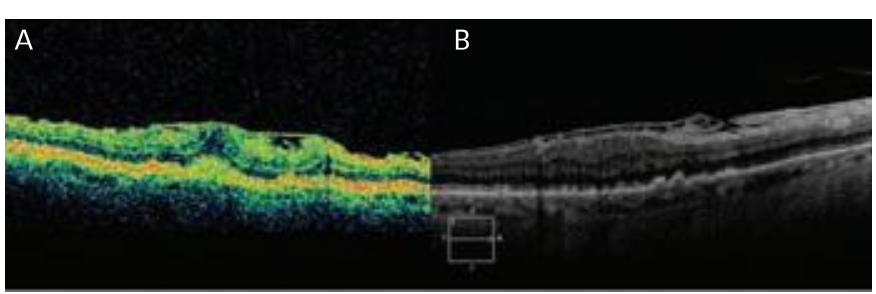

A: Exudative age-related macular degeneration (AMD) shown using time-domain optical coherence tomography (OCT). B: Dry AMD shown using spectral-domain OCT.

Figure 7: Illustration of Reactive Gliosis in Age-related Macular Degeneration

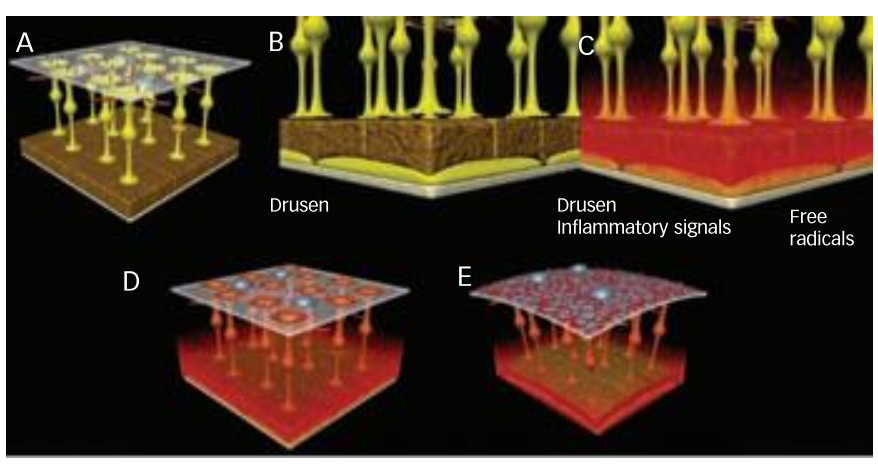

A: The normal retinal microarchitecture highlighting only the retinal pigment epithelium (RPE) (brown), Müller cells (yellow), astrocytes (silver), retinal blood vessels (red) and inner limiting membrane (ILM) (transparent grey). B: Dry AMD with a close-up of drusen accumulation under the RPE and the proximity of Müller cells (yellow) over the RPE. C: Drusen inflammatory and oxidative stress (red staining of RPE and Müller cells) stimulus. D: Consequent reactive gliosis with Müller cells (orange) and astrocytes migration towards the ILM vitreal side. E: Epiretinal membrane formation in dry AMD.

Figure 8: Time-domain Optical Coherence Tomography showing the Presence of a Macular Hole and Choroidal Neovascularisation in Age-related Macular Degeneration

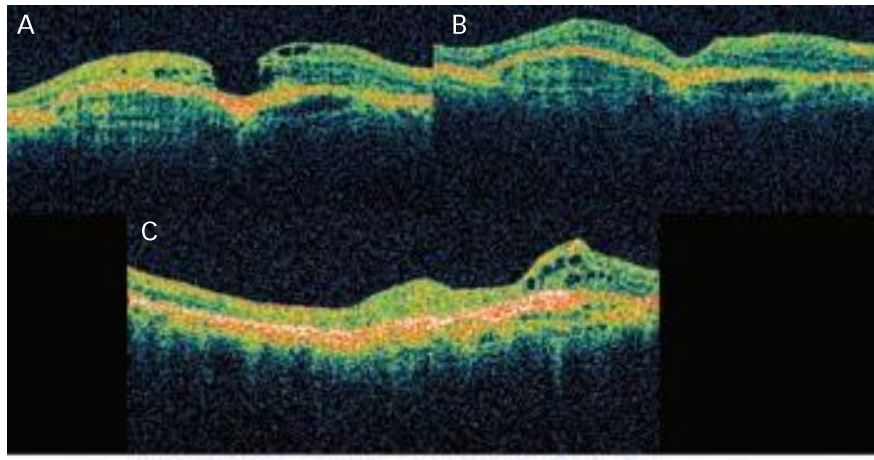

A: Macular hole and choroidal neovascularisation (CNV) in age-related macular degeneration (AMD). B: After vitrectomy and inner limiting membrane (ILM) peeling, the macular hole closed. C: However, despite the effects of higher oxygen availability to the retina post-vitrectomy the CNV reactivated and more frequent anti-vascular endothelial growth factor (VEGF) injections were needed compared to before the vitrectomy.

and bevacizumab have been used as first line treatments in combined cases of AMD and VMT, although extended treatment is warranted to control exudative recurrences (see Figure 8 8). $^{17}$ Interestingly, traction often worsens clinically and on imaging following intravitreal injections (IVI) and even macular holes have been described after anti-VEGF injection for exudative AMD. ${ }^{67}$ If eyes remain refractory to treatment despite IVI treatments, Mojana and colleagues recommended a surgical release of the traction. They performed surgery in five patients 
Figure 9: Subfoveal Classic Choroidal Neovascularisation Post-epiretinal Membrane Peeling in Dry Age-related Macular Degeneration

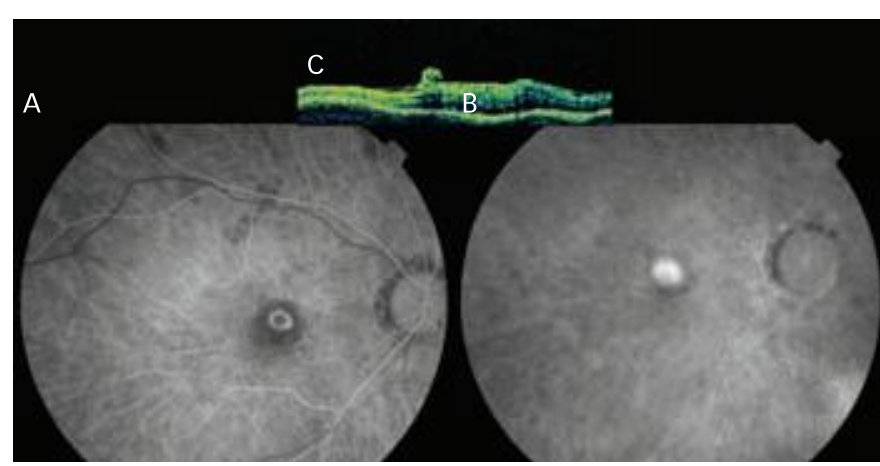

A, B: Classic choroidal neovascularisation (CNV); C: post-epiretinal membrane (ERM) (time-domain optical coherence tomography [OCT] image) peeling in dry age-related macular degeneration (AMD).

Figure 10: latrogenic Intra-operative Retinal Pigment Epithelium Tears during 25-gauge Epiretinal Membrane Peeling

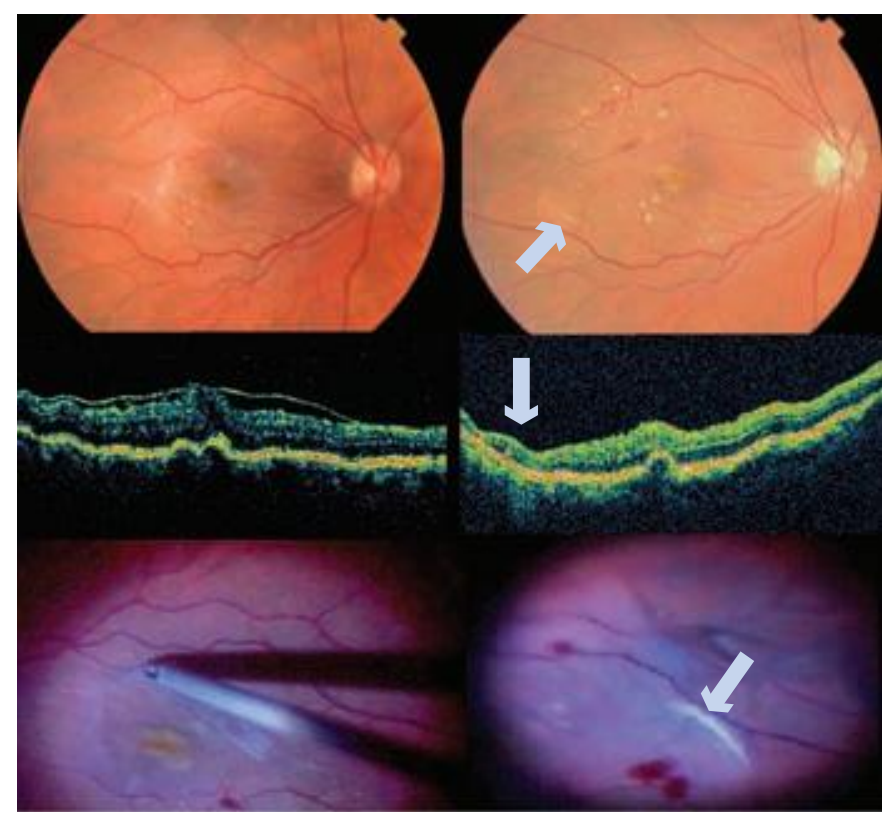

The tears are indicated by arrows.

with persistent VMT, which they proposed is a mechanism that results in patients presenting with pharmacologic-resistant disease, possibly through tractional forces antagonising and limiting drug access to areas of macular disease. ${ }^{12,17}$ Others suggest that if vision improves with IVI alone, no further surgical steps need be taken to address VMT if the benefits offered in tackling the latter do not outweigh the risks or if chronic atrophic changes have already set in. In all instances, spontaneous resolution of VMT from IVI treatment or a PVD is rare. ${ }^{68}$

\section{Vitreolysis}

A future adjuvant therapy for tractional maculopathies in the presence of a partial PVD is the use of proteolytic human enzymes against the vitreoretinal protein matrix. An ideal pharmacological agent is one that induces complete liquefaction and dehiscence of the vitreous simultaneously. ${ }^{4}$ Plasmin, a serine protease that can hydrolyze laminin and fibronectin glycoproteins at the vitreoretinal interface, has been suggested. More recently, ocriplasmin, a recombinant product containing only the catalytic domain of human plasmin has garnered interest, as it is molecularly more stable and easier to store and administer than its predecessor. This vitreolytic molecule has shown promise through the MIVI-I (Microplasmin intravitreal administration in patients with vitreomacular traction scheduled for vitrectomy) and MIVI-IIT (intravitreal injection of microplasmin for treatment of vitreomacular adhesion: results of a prospective randomised sham-controlled Phase II) trials and should soon be available; however, further research is needed as failure of complete chemical vitreolysis can result in anomalous vitreous dynamics and vitreoschisis, putting the eye at an increased risk for complications. ${ }^{69-71}$

\section{Exudative Age-related Macular Degeneration Progression following Tractional Maculopathy}

In a few case reports, authors have established a clear sequence that CNV might develop post-ERM or macular hole repair. Two types of cause are proposed. In non-surgical cases, the CNV develops owing to the natural history of tractional disease, with subretinal fluid inducing inflammatory cytokines and mediators leading to Bruch's membrane disruption. In surgical cases, despite the theoretical benefits of a vitrectomy inducing liberation of macular traction and increase in oxygen tension, it appears that post-operative trauma and the surgical sequelae on the RPE may overpower the beneficial effects of surgery to incite neovascularisation. ${ }^{72-79}$ This was the case in the authors small gauge vitrectomy series as described above, where the sole patient whose visual acuity did not improve was due to the result of development of de novo CNV post 25-ga vitrectomy for ERM removal (see Figure 9).

Management techniques advocate the sequential treatment of the CNV first followed by surgical hole repair (see Figure 8). The posited reasoning is that pharmacological involution of CNV might cause macular hole edges to regress. With these techniques and improved surgical instrumentation, improved visual outcomes can be expected. ${ }^{76}$ Photodynamic therapy in conjunction with vitrectomy has also been reported as a successful treatment, despite the evolution of anti-angiogenesis treatments. ${ }^{75}$

\section{Post-operative Complications}

The surgical complications are the same as for general vitrectomy, and include intraoperative retinal breaks (5\%), intraretinal bleeding after tractional membrane removal and progressive nuclear sclerosis (12-68 \%), retinal detachment (5\%), macular oedema, retinal pigment epitheliopathy and recurrence of ERM (10\%). ${ }^{58}$ Submacular surgery in exudative AMD with forceful injection of subretinal tissue plasminogen activator (t-PA) has also been reported to induce occasionally an iatrogenic macular hole..$^{80}$ However, with combined anti-VEGF and vitrectomy treatment for exudative AMD and tractional maculopathies, even if visual acuity improves and traction rarely recurs if surgery is successful, chronic irreversible RPE changes could have already set in. Most patients with exudative AMD require repeated and more frequent anti-VEGF injections despite release of tractional pathologies through vitrectomy.

\section{Retinal Pigment Epithelium Detachment and Tears}

RPE tears and detachments in AMD have been described post-anti-VEGF IVI. Proposed mechanisms include the possibility of rupture of a previously present RPE detachment (PED), an iatrogenic tear from blunt needle insertion, vitreous incarceration causing VMT and vascular 
membrane contraction, or the modulatory changes following the properties of the anti-angiogenic drug itself. All of these remain to be proven. ${ }^{78,81,82}$ Spontaneous RPE tears in patients with AMD have been previously reported in the literature, indicating the inherent fragility of this degenerated retinal tissue. Meyer and Toth suggested that chronic weak traction at the vitreomacular interface induces excess shear and tangential forces applied and transmitted to the RPE, leading to mechanisms that make the RPE more susceptible to RPE detachment and tears, and even secondary macular holes. ${ }^{83-85}$ In addition, Chan and colleagues linked PED and RPE tears in a study, where 2,785 injections in 1,064 patients demonstrated an incidence of $2.22 \%$ RPE tears among all AMD eyes and $17.5 \%$ among eyes with previous PED..$^{86}$ However, RPE tears after ERM removal in patients with $A M D$, as seen in two of our own surgical AMD cohort, have previously been unreported (see Figure 10).

Previous authors have observed that spontaneous RPE tear can occur in patients with AMD secondary to the underlying PED. ${ }^{87}$ The authors suggest that in the absence of obvious predisposing factors, Müller cells and reactive gliosis are an important aetiological component by coupling inner and outer retina movements and mechanotransduction forces (see Figure 11).

In AMD, Müller cells can directly form ERMs. Surgical removal or VMT on the ERM might directly transmit these forces along all retinal layers to create tears on the underlying photoreceptor-RPE junction. Given the similar composition of the vitreoretinal and the photoreceptor-RPE interface, one can assume that abnormalities in one can directly affect the other. ${ }^{88}$ By using atomic force microscopy to measure Müller cell elasticity in different underlying substrates in vitro, the authors demonstrated that Müller cells increase their mechanical stiffness 10-fold when grown on a stiff substrate, such as the ageing Bruch's membrane in AMD (data not shown). It is hypothesised that these biophysical phenomena can account for the creation of an RPE tear in that a mechanically stiffer Müller cell can support the transmission of force from the internal limiting membrane to the outer retina and RPE (see Figure 11). The intercalation of Müller cells into adjacent photoreceptor structures predisposes them to the transmission of tractional forces so that when surgical instruments pull on the glial epiretinal and VMA elements, the same force can be transmitted to the outer retina, directly damaging the already weakened and atrophic RPE. Therefore, extreme care must be exercised when performing ERM and ILM peeling in patients with $A M D$, and RPE tears should be recognised as a possible direct iatrogenic complication of these procedures in patients with dry or exudative AMD (see Figure 10).

\section{Figure 11: Illustration of Epiretinal Membrane Peeling and Secondary latrogenic Retinal Pigment Epithelium Tear in Age-related Macular Degeneration}

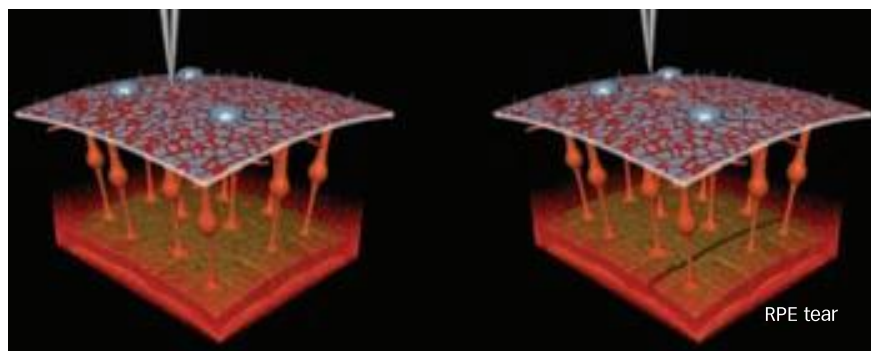

The reactive Müller cells in age-related macular degeneration (AMD) can migrate towards the RPE, creating a potential transmission of forces from the inner limiting membrane (ILM) vitreal surface during epiretinal membrane (ERM) peeling, generating enough retinal pigment epithelium (RPE) traction to cause it to tear.

\section{Conclusion}

Superior imaging techniques and advanced biochemical analysis have provided clinicians and researchers with new understandings of the biomechanics of the vitreomacular interface. There is now no longer a strict separation of the different aetiologies of disease. It is now possible to regard AMD and VMT pathologies as concurrent diseases that affect each other. At an early phase, partial anomalous PVD are often found in the presence of non-exudative AMD. With time and chronic traction, this can cause the AMD to degenerate into an exudative form, which in turn can trigger reactive gliosis, which will make the tractional disease worse. In addition, other environmental, genetic and inflammatory factors are likely to have a large role in AMD pathogenesis. ${ }^{17}$ These data should be considered once ocriplasmin is licensed for use.

With so many factors in play, possible future directions in the targeting of $\mathrm{AMD}$ and tractional maculopathies are limitless. Vitreous gel biomechanics and Müller cell gliotic reaction are excellent future targets for research. ${ }^{35}$ Controlling the potassium channels of Müller cells might also have a part in decreasing macular oedema (as corticosteroids can stimulate fluid clearance by Müller cells). ${ }^{50}$

It would be interesting to know whether future imaging techniques will allow one to detect early and late AMD vitreomacular changes so as to detect early damage and follow cellular responses to treatment or even modulate and curb progression through the potential control of reactive gliosis. Already, findings relating to the role of melatonin in reactive gliosis regulation and opticin as a possible vitreomacular interface binding protein have garnered interest. ${ }^{89,90}$ This gives researchers hope for catching AMD pathologies early on instead of treating their downstream consequences
1. Zarbin MA, Current concepts in the pathogenesis of age-related macular degeneration, Arch Ophthalmol, 2004;122(4):598-614.

2. Penfold PL, Madigan MC, Gillies MC, Provis JM, Immunological and aetiological aspects of macular degeneration, Prog Retin Eye Res, 2001;20(3):385-414.

3. Bishop PN, Holmes DF, Kadler KE, et al., Age-related changes on the surface of vitreous collagen fibrils, Invest Ophthalmol Vis Sci, 2004;45(4):1041-6.

4. Sebag J, Anomalous posterior vitreous detachment: a unifying concept in vitreo-retinal disease, Graefes Arch Clin Exp Ophthalmo 2004;242(8):690-8

5. Krebs I, Glittenberg C, Zeiler F, Binder S, Spectral domain optical coherence tomography for higher precision in the evaluation of vitreoretinal adhesions in exudative age-related macular degeneration, Br J Ophthalmol, 2011:95(10):1415-8.

Mirza RG, Johnson MW, Jampol LM, Optical coherence tomography use in evaluation of the vitreoretinal interface: a review, surv ophthalmol, 2007:52(4):397-421.

Georgalas I, Heatley C, Ezra E, Retinal pigment epithelium detachment associated with vitreomacular traction syndrome
A case report, Int Ophthalmol, 2009;29(5):431-3.

8. Krebs I, Brannath W, Glittenberg C, et al., Posterior vitreomacular adhesion: a potential risk factor for exudative age-related macular degeneration? Am I Ophthalmol, 2007;144(5):741-6.

9. Robison $\mathrm{CD}$, Krebs I, Binder S, et al., Vitreomacular adhesion in active and end-stage age-related macular degeneration Am I Ophthalmol, 2009:148(1):79-82

10. Jun Lee $\mathrm{S}$, Lee CS, Jun Koh $\mathrm{H}$, Posterior vitreomacular adhesion and risk of exudative age-related macular degeneration: paired eye study, Am J Ophthalmol, 2009;147(4):621-6.

11. Weber-Krause $B$, Eckardt $U$, Incidence of posterior vitreous detachment in eyes with and without age-related macular degeneration. An ultrasonic study, Ophthalmologe $1996 ; 93(6): 660-5$.

12. Mojana F, Cheng L, Bartsch D-UG, et al., The role of abnorma vitreomacular adhesion in age-related macular degeneration: spectral optical coherence tomography and surgical results, Am I Ophthalmol, 2008:146(2):218-27.

13. Ondes F, Yilmaz G, Acar M, et al., Role of the vitreous in age-related macular degeneration, Jpn J ophthalmo 2000;44(1):91-3.

14. Rezende F, Kickinger M, Prado R, Vitreomacular and photoreceptor-retinal pigment epithelium interface relationships in age-related macular degeneration, Invest ophthalmol Vis Sci, 2010;51(E-abstract 520).

15. Krebs I, Glittenberg C, Zeiler F, Binder S, Spectral domain optical coherence tomography for higher precision in the evaluation of vitreoretinal adhesions in exudative age-related macular degeneration, Br J Ophthalmol, 2011;95(10):1415-8.

16. Schulze $S$, Hoerle $S$, Mennel $S$, Kroll $P$, Vitreomacular traction and exudative age-related macular degeneration, Acta Ophthalmol, 2008;86(5):470-81.

17. Rotsos T, Sagoo MS, daCruz L, et al., Intravitreal anti-VEGE treatment in eyes with combined choroidal neovascularisation and vitreomacular traction syndrome, Br J Ophthalmol, 2010;94(9):1205-10

18. Spaide R, Armstrong D, Browne R, Choroidal neovascularization in age-related macular degeneration what is the cause?, Retina, 2003;23(5):595-614 
19. Kimura H, Kuroda S, Nagata M, Premacular cortical vitreous in patients with a rhegmatogenous retinal detachment, Retina, 2004;24(2):329-30

20. Sullivan R, Penfold P, Pow DV, Neuronal migration and glial remodeling in degenerating retinas of aged rats and in nonneovascular AMD, invest Ophthalmol Vis Sci, 2003:44(2):856-65.

21. Limb GA, Daniels JT, Pleass R, et al., Differential expression of matrix metalloproteinases 2 and 9 by glial Müller cells: response to soluble and extracellular matrix-bound tumor necrosis factor-alpha, Am J Pathol, 2002;160(5):1847-55.

22. Bringmann $A$, Francke $M$, Reichenbach $A$, Müller cells in retinopathies. In: Leif $\mathrm{H}$, ed. Advances in Molecular and Cell Biology, 2003:31:1117-32

23. Newman E, Reichenbach A, The Müller cell: a functional element of the retina, Trends Neurosci, 1996:19(8):307-12.

24. Puro D, Growth factors and Müller cells, Prog Retin Eye Res, 1995; 15:89-101

25. Dietrich $P$, Walker $P$, Saas $P$, Death receptors on reactive astrocytes: a key role in the fine tuning of brain inflammation? Neurology, 2003;60(4):548-54.

26. Pekny M, Nilsson M, Astrocyte activation and reactive gliosis, Glia, 2005;50(4):427-34.

27. Malgorzata Goczalik I, Raap M, Weick M, et al., The activation of IL-8 receptors in cultured guinea pig Müller glial cells is modified by signals from retinal pigment epithelium, J Neuroimmunol, 2005:161(1):49-60.

28. Donoso LA, Kim D, Frost A, et al., The role of inflammation in the pathogenesis of age-related macular degeneration, surv Ophthalmol, 51(2):137-52

29. Caicedo A, Espinosa-Heidmann DG, Piña Y, et al., Bloodderived macrophages infiltrate the retina and activate Müller glial cells under experimental choroidal neovascularization, Exp Eye Res, 2005;81(1):38-47.

30. Harada C, Mitamura Y, Harada T, The role of cytokines and trophic factors in epiretinal membranes: involvement of signa transduction in glial cells, Prog Retin Eye Res, 2006;25(2):149-64.

31. Fischer $A$, Omar $G$, Eubanks J, et al., Different aspects of gliosis in retinal Muller glia can be induced by CNTF, insulin, and FGF2 in the absence of damage, Mol Vis, 2004;22(10):973-86.

32. Kubay OV, Charteris DG, Newland HS, Raymond GL, Retina detachment neuropathology and potential strategies for neuroprotection, Surv Ophthalmol, 2005;50(5):463-75

33. Eichler W, Yafai Y, Wiedemann P, Reichenbach A, Angiogenesis-related factors derived from retinal glial (Muller) cells in hypoxia, NeuroReport, 2004;15(10):1633-7.

34. Hageman GS, Luthert PJ, Victor Chong NH, et al., An integrate hypothesis that considers drusen as biomarkers of immunemediated processes at the RPE-Bruch's membrane interface in aging and age-related macular degeneration, Prog Retin Eye Res, 2001;20(6):705-32.

35. Bringmann A, Pannicke T, Grosche J, et al., Müller cells in the healthy and diseased retina, Prog Retin Eye Res, 2006;25(4):397-424

36. Sofroniew MV, Reactive astrocytes in neural repair and protection, Neuroscientist, 2005;11(5):400-7.

37. Nakazawa T, Takeda M, Lewis GP, et al., Attenuated glia reactions and photoreceptor degeneration after retinal detachment in mice deficient in glial fibrillary acidic protein and vimentin, Invest Ophthalmol Vis Sci, 2007:48(6):2760-8.

38. Sofroniew M, Vinters $H$, Astrocytes: biology and pathology, Acta Neuropathol, 2010;119(1):7-35.

39. Kielian T, Esen N, Effects of neuroinflammation on glia-glia gap junctional intercellular communication: a perspective, Neurochem Int, 2004;45(2-3):429-36.

40. Pitz S, Moll R, Intermediate-filament expression in ocular tissue, Prog Retin Eye Res, 2002;21(2):241-62.

41. Guidry C, Medeiros NE, Curcio CA, Phenotypic variation of retinal pigment epithelium in age-related macular degeneration, Invest Ophthalmol Vis Sci, 2002:43(1):267-73.

42. Dyer MA, Cepko $\mathrm{CL}$, Regulating proliferation during retinal development, Nat Rev Neurosci, 2001;2(5):333-42.

43. Johnson PT, Lewis GP, Talaga KC, et al., Drusen-associated degeneration in the Retina, Invest Ophthalmol Vis Sci, 2003;44(10):4481-8.
44. Fisher SK, Lewis GP, Müller cell and neuronal remodeling in retinal detachment and reattachment and their potential consequences for visual recovery: a review and reconsideration of recent data, Vis Res, 2003;43(8):887-97.

45. Ramírez JM, Ramírez Al, Salazar JJ, et al., Changes of astrocytes in retinal ageing and age-related macular degeneration, Exp Eye Res, 2001;73(5):601-15.

46. Vázquez-Chona $F$, song BK, Geisert EE, Temporal changes in gene expression after injury in the rat Retina Invest Ophthalmol Vis Sci, 2004;45(8):2737-46

47. Yamada N, Kishi S, Tomographic features and surgical outcomes of vitreomacular traction syndrome, Am I Ophthalmo 2005;139(1):112-7

48. Guidry C, Isolation and characterization of porcine Müller cells. Myofibroblastic dedifferentiation in culture, Invest Ophthalmol Vis Sci, 1996;37(5):740-52.

49. Guidry C, Bradley KM, King JL, Tractional force generation by human Müller cells: growth factor responsiveness and integrin receptor involvement, Invest ophthalmol Vis Sci, 2003;44(3):1355-63.

50. Reichenbach A, Wurm A, Pannicke T, et al., Müller cells as players in retinal degeneration and edema, Graefes Arch Clin Exp Ophthalmol, 2007:245:627-36.

51. Krebs I, Hagen $S$, Haas $P$, et al., The vitreo-retinal interface in macular diseases, Spektrum Augenheilkunde, 2009;23(1):2-11.

52. Wilkins J, Puliafito C, Hee M, et al., Characterization of epiretinal membranes using optical coherence tomography, Ophthalmology, 1996:103(12):2142-51.

53. Mori K, Gehlbach P, Sano A, et al., Comparison of epiretin membranes of differing pathogenesis using optical coherence tomography, Retina, 2004;24(1):57-62.

54. Witherspoon C, Morris R, Fivgas G, et al., Internal limiting membrane removal in the management of idiopathic macular hole. Investigative Ophthalmology and Visual Science, Fort Lauderdale, FL, 1999 Meeting Program, S114.

55. Smiddy WE, Flynn HW, Jr, Pathogenesis of macular holes and therapeutic implications, Am J Ophthalmol, 2004:137(3):525-37.

56. Schumann RG, Schaumberger MM, Rohleder M, et al Ultrastructure of the vitreomacular interface in full-thickness idiopathic macular holes: a consecutive analysis of 100 cases, Am J Ophthalmol, 2006;141(6):1112-9.

57. Kumar A, Wagh V, Prakash $G$, et al., Visual outcome and electron microscopic features of indocyanine green-assisted internal limiting membrane peeling from macular hole of various aetiologies, Indian J ophthalmol, 2005;53(3):159-65.

58. Falkner-Radler $\mathrm{Cl}$, Binder $\mathrm{S}$, Age-related macular degeneration III: epiretinal membranes. In: Cavallotti CAP, Cerulli L (eds), Aging Medicine, New York: Humana Press; 2008:265-71

59. Messmer EM, Heidenkummer H-P, Kampik A, Ultrastructure of epiretinal membranes associated with macular holes, Graefes Arch Clin Exp Ophthalmol, 1998;236(4):248-54.

60. Ito $Y$, Terasaki H, Suzuki T, et al., Mapping posterior vitreous detachment by optical coherence tomography in eyes with idiopathic macular hole, Am J Ophthalmol, 2003;135(3):351-5.

61. Ikeda T, Sawa H, Koizumi K, et al., Pars plana vitrectomy for regression of choroidal neovascularization with age-related macular degeneration, Acta Ophthalmol Scand, 2000;78(4):460-4

62. Schmidt JC, Mennel S, Hörle S, Meyer $\mathrm{CH}$, High incidence of vitreomacular traction in recurrent choroidal neovascularisation after repeated photodynamic therapy, $\mathrm{Br}$ ophthalmol, 2006;90(11):1361-2.

63. Kuhn F, Point: To peel or not to peel, that is the question, Ophthalmology, 2002;109(1):9-11.

64. Falkner-Radler $\mathrm{Cl}$, Binder $\mathrm{S}$, Age-related macular degeneration II: idiopathic macular holes. In: Cavallotti CAP, Cerulli L (eds), Aging Medicine, New York: Humana Press; 2008:257-64.

65. Roller AB, Mahajan VB, Boldt HC, et al., Effects of vitrectomy on age-related macular degeneration, ophthalmology, 2010;117(7):1381-6.

66. Rezende $F$, Vitreoretinal surgery for tractional maculopathies in age-related macular degeneration, American Society of Retina Specialists Conference, Vancouver, September 1 2010:12.

67. Querques G, Souied EH, Soubrane G, Macular hole following intravitreal ranibizumab injection for choroidal neovascula membrane caused by age-related macular degeneration, Acta Ophthalmol, 2009;87(2):235-7.

68. Rodríguez A, Infante R, Rodríguez F, Valencia M, Spontaneous separation in idiopathic vitreomacular traction syndrome associated with contralateral full-thickness macular hole, Eur J Ophthalmol, 2006;16(5):733-40

69. de Smet MD, Gandorfer A, Stalmans P, et al., Microplasmin intravitreal administration in patients with vitreomacula traction scheduled for vitrectomy: the MIVI I Trial, Ophthalmology, 2009;116(7):1349-55.

70. Stalmans P, Delaey C, de Smet MD, et al., Intravitreal injection of microplasmin for treatment of vitreomacular adhesion: results of a prospective, randomized, sham-controlled phase ii trial (The MIVI-IIT Trial), Retina, 2010;30(7):1122-7.

71. Rhéaume M-A, Vawas D, Pharmacologic vitreolysis, Semin ophthalmol, 2010:25(5-6):295-302.

72. Holekamp NM, Shui Y-B, Beebe DC, Vitrectomy surgery increases oxygen exposure to the lens: a possible mechanism for nuclear cataract formation, Am J Ophthalmol 2005;139(2):302-10

73. Stefánsson E, Physiology of vitreous surgery, Graefes Arch Clin Exp Ophthalmol, 2009;247(2):147-63

74. Spies A, Messner LV, An untreated macular hole with adjacent choroidal neovascularization, Optom Vis Sci, 2003:80(9):619-22.

75. Recchia FM, Williams GA, Visual improvement following sequential photodynamic therapy and vitrectomy for coexisting macular hole and choroidal neovascularization, Retina, 2003:23(1):110-3.

76. Singh RP, Kaiser PK, Treatment of co-existent occult choroidal neovascular membrane and macular hole, surv Ophthalmol, 2007;52(5):547-50.

77. Elsing SH, Postel EA, Gill MK, et al., Management of eyes with both idiopathic macular hole and choroidal neovascularization, Retina, 2001;21(6):613-8.

78. Tabandeh $\mathrm{H}$, Smiddy WE, Choroidal neovascularization following macular hole surgery, Retina, 1999;19(5):414-7.

79. Natarajan S, Mehta H, Mahapatra S, Sharma S, A rare case of choroidal neovascularization following macular hole surgery, Graefes Arch Clin Exp Ophthalmol, 2006;244(2):271-3.

80. Bakri S, Sears J, Lewis H, Management of macular hole and submacular hemorrhage in the same eye, Graefes Arch Clin Exp Ophthalmol, 2007;245(4):609-11.

81. Warden SM, Pachydaki SI, Christoforidis JB, et al., Choroidal neovascularization after epiretinal membrane removal, Arch Ophthalmol, 2006;124(11):1652-4.

82. Bakri SJ, Kitzmann AS, Retinal pigment epithelial tear after intravitreal ranibizumab, Am J Ophthalmol, 2007;143(3):505-7.

83. Meyer $\mathrm{CH}$, Mennel S, Schmidt JC, Kroll P, Acute retinal pigment epithelial tear following intravitreal bevacizumab (Avastin) injection for occult choroidal neovascularisation secondary to age related macular degeneration, Br J Ophthalmol, 2006;90(9):1207-8.

84. Meyer C, Toth C, Retinal pigment epithelial tear with vitreomacular attachment: a novel pathogenic feature Graefes Arch Clin Exp Ophthalmol, 2001;239(5):325-33.

85. Emerson GG, Ghazi NG, Spontaneous rip of the retinal pigment epithelium with a macular hole in neovascular age-related macular degeneration, Am J Ophthalmol, 2005;140(2):316-8.

86. Chan C, Meyer C, Gross J, et al., Retinal pigment epithelial tears after intravitreal bevacizumab injection for neovascular age-related macular degeneration, Retina, 2007:27(5):541-51.

87. Lafaut BA, Aisenbrey S, Vanden Broecke C, et al., Clinicopathological correlation of retinal pigment epithelial tears in exudative age related macular degeneration: pretear, tear, and scarred tear, Br J Ophthalmol, 2001;85(4):454-60.

88. Quranta-El Maftouhi M, Mauget-Faysse M, Anomalous vitreoretinal adhesions in patients with exudative age-related macular degeneration: an OCT study, Eur J Ophthalmol, 2006:16(1):134-7.

89. Ramesh S, Bonshek RE, Bishop PN, Immunolocalisation of opticin in the human eye, Br J Ophthalmol, 2004:88(5):697-702.

90. Baydas G, Ozer M, Yasar A, et al., Melatonin prevents oxidative stress and inhibits reactive gliosis induced by hyperhomocysteinemia in rats, Biochemistry, 2006;71(0):S91-5. 